\section{Tiny contributors to severe obesity inside the gut}

\author{
Yolanda Sanz 두, Marta Olivares
}

Obesity is a key player in the current global health crisis, acting as a leading risk factor in the main causes of morbidity and mortality, ranging from cardiovascular disease (CVD) and type 2 diabetes (T2D) to respiratory illnesses. ${ }^{1}$ While unhealthy diets and sedentary lifestyles together with polygenetic risks represent major causes of obesity, recent studies suggest that the gut microbiota also plays a part. ${ }^{2}$ A large body of evidence has revealed alterations in the gut microbiota composition and function of obese subjects compared with healthy controls. ${ }^{3-5}$ Moreover, certain prospective observational and interventional studies have identified microbiome signatures that precede the onset of obesity $^{67}$ or influence the response to diet. ${ }^{8}$ Notwithstanding, precise identification of microbiome biomarkers is difficult due to the large interindividual variability of the microbiota as well as of the host and environmental influential factors and the lack of methodological standards, all of which hinder progress towards assessing their causal role in obesity. To date, reduced microbial gene richness and diversity are the most consistent features associated with vulnerability to obesity $^{6}$ and the severity. ${ }^{3-5}$ Metabolites generated by the gut microbiota or hostmicrobe co-metabolic processes might also play detrimental or protective roles in obesity and associated complications (for review ${ }^{9}$ ). These include trimethylamine oxide, for instance, which is produced from dietary choline and carnitine by the host's gut microbial and liver enzymes and is linked to CVD risk and insulin resistance. Imidazole propionate, which results from histidine catabolism, is involved in insulin resistance and T2D. Branched-chain amino acids and indole derivatives (eg, 3-indolepropionic acid) are linked to insulin resistance or gut barrier protection and insulin secretion and sensitivity, respectively. Short-chain fatty acids, especially butyric acid, are linked to the enhanced action of incretins in satiety, insulin sensitivity and glucose tolerance.

Indeed, the gut is the habitat of trillions of microorganisms, acting as a first line of

Microbial Ecology, Nutrition and Health, IATA-CSIC, Valencia, Spain

Correspondence to Dr Yolanda Sanz, Institute of Agrochemistry and Food Technology, Spanish National Research Council (IATA-CSIC), 46980 Paterna-Valencia, Spain; yolsanz@iata.csic.es defence against the impact of unhealthy dietary exposure and as diet-health mediators, affecting metabolic, endocrine, immune and neural pathways that regulate interorgan communication and whole body metabolism. ${ }^{10}$ Disentangling how these complex interactions work is essential to pinpoint the causal mechanisms negatively affecting the metabolic phenotype and to identify more precise strategies to intercept the circular cascade of disease triggers and thus improve obesity management options.

In Gut, Belda et al take on the challenge of identifying the mechanisms through which alterations in the gut microbiota can be functionally involved in severe obesity, applying a translational research approach and validating the findings in different study models. ${ }^{11}$ Overall, the results point to the role played by the low abundance of microbial genes capable of supplying biotin to the host, which is compatible with the suboptimal biotin status and hallmarks of metabolic dysfunction and inflammation detected in the affected subjects. ${ }^{11}$

The human host's primary source of biotin comes from the diet but gut bacteria can also synthetise biotin and, thus, presumably contribute to the host's biotin status. Biotin acts as enzyme cofactor and, for example, participates in the carboxylation of pyruvate to oxaloacetate and triggers fatty-acid biosynthesis, contributing to adipose tissue and whole body metabolism. ${ }^{12}$

Biotin deficiency has been described in both obesity and T2D, moreover, biotin supplementation has been shown to improve patients' glucose metabolism. ${ }^{13}$ In Gut, Belda et al have progressed beyond these initial observations and are the first authors to correlate the severity of obesity (body mass index $>35-40 \mathrm{~kg} / \mathrm{m}^{2}$ ) with lowered gut bacterial cell numbers and microbial genes potentially involved in biotin metabolism. ${ }^{11}$ This was done by performing a cross-sectional analysis of the faecal microbiota of around 1500 subjects from the MetaCardis cohort and making comparisons after transforming relative data on the abundance of metagenes into absolute data by quantifying total microbial cells. Specifically, the study shows a decrease in genes involved in the biosynthesis and uptake of biotin as well as a reduction of microbial biotin producers (biotin prototrophs dominated by taxa belonging to Proteobacteria and Bacteroidetes) and consumers (biotin auxotrophs dominated by taxa belonging to Firmicutes). Ideally, the findings should have been supported with results from functional omics analysis to predict the real metabolic capacities of the microbial community more accurately. Even so, previous genomic and in vitro studies are in line with Belda et al's findings, showing that the most abundant butyrate producers of the phylum Firmicutes have multiple vitamin auxotrophies and their ability to thrive in the gut ecosystem might partly depend on vitamins supplied by other microbial community members. ${ }^{14}$

In contrast to Belda et al, previous studies have reported an increased abundance of genes potentially involved in biotin biosynthesis in subjects with obesity and T2D, based on comparisons of relative abundance of metagenes, ${ }^{13}$ rather than on an absolute quantification. Therefore, the study published in Gut also invites us to revisit previous dogmas and studies intending to spot microbiome signatures of obesity and/ or T2D, such as Bacteroides-enriched microbiomes, without considering cell loads and their impact on the microbiome functions. ${ }^{3}$

Consistent with the first findings in subjects with severe obesity, Belda et al also showed that bariatric surgery, in both humans and animals, led to increased microbial biotin producers and an improved host biotin status. $^{11}$

Moreover, the authors have made progress in the consolidation of their hypothesis by carrying out an animal study of high-fat diet (HFD)-induced obesity, which reduced the microbial counts and biotin gene potential as well as the host's biotin levels. However, this study did not prove causality as the effects on biotin could be due to either diet or dietinduced dysbiosis, or indeed both. In fact, obesity and obesogenic diet-induced intestinal inflammation could be directly responsible for biotin status impairment through alterations in biotin-transporter expression and absorption. ${ }^{15}$ In turn, biotin deficiency could also theoretically impair immunity and contribute to inflammation. ${ }^{15}$

The authors made additional murine studies to address the main question of whether the reduced potential capacity of the microbiota to supply biotin was causally linked to the host's poor biotin status or whether it was merely a consequence of the reduced bacterial cell counts reported in severe obesity. Studies performed in germ-free (GF), antibiotic-treated specific pathogen-free mice and, particularly, via faecal microbiota transfers from humans to mice, provide more definitive conclusions. Both the absence of microbiota in GF chow-fed mice and the reduction of 
microbial loads due to antibiotic treatment reduced their serum biotin levels, supporting a causal role for the microbiota in supplying $\mathrm{B}$ vitamins to the host. Definitively, transferring the human microbiota from two subjects with severe obesity, on the one hand, and two lean subjects who harboured increased amounts of bacterial biotin producers, on the other, showed that both the Western diet and the obese microbiota in chow-fed individuals played a causal role in the biotin status of mice. It is not possible to extrapolate this finding to severe obesity since the transfer of microbiota was performed intentionally with subjects having specific microbiome features; however, the selection serves the purpose of the study and the results support the author's hypothesis. These findings also invite one to consider the need to progress in this research arena, and facilitate the use of the individual's microbiome data for stratification and personalisation of diagnostic, prognostic and therapeutic approaches. Indeed, individualisation is important as not all subjects with severe obesity show reduced microbial gene richness, which may range from $40 \%$ to $75 \% .{ }^{511}$ It is noteworthy that the Western or HFD diet exerted the greatest effect on biotin status and, although the dietary regimen exemplifies an extreme situation, the results also point to the prominent role of the diet in the malnourished obese phenotype.

Furthermore, the authors explored the clinical applicability of the new knowledge. To do so, they administered oral biotin and a prebiotic (fructo oligosaccharides) to mice with HFD-induced obesity. Results showed improvements in body weight, glucose metabolism and biotin status, as well as microbial diversity and functional capacity for biotin production. Of special interest are the results for the FOS-treated mice, in which the relative abundance of biotin producers increased with a simultaneous reduction in biotin transporters (auxotrophs). This finding, compared with human studies, strongly supports the idea that gut bacteria contribute to supplying biotin to the host. However, it must still be established whether the prebiotic effect on biotin status is a consequence of its anti-obesogenic effects or a consequence of the induced microbiota changes.

A key implication of the paper is the potential translation of this knowledge into practice, considering the host's biotin status and microbiota profile in the patientstratification process, as well as microbiota modulators for the clinical management of severe obesity. Nonetheless, further evidence of the efficacy of interventions directed at the gut microbiota in humans is needed to confirm this hypothesis.

Overall, the study elegantly exemplifies how to gain a mechanistic understanding of the microbiota's role in specific conditions and to confirm hypotheses generated in large observational studies through the parallel use of models able to prove cause-effect relationships.

The study also raises many other questions: Which comes first, obesity or a microbiome profile deficient in biotin-producing genes? Do bacterial auxotrophs/potential consumers and prototrophs/potential producers interact and affect the human host's vitamin supply? Does microbiome biosynthesis potential differ in the large and small intestines considering that biotin absorption mainly takes place in the jejunum? Can the effects of dietary intervention (prebiotic) be translated to humans, considering the variability of their microbiota and the individualised responses to fibres as well as the diverse obese phenotypes?

\section{Twitter Yolanda Sanz @YolsanzSanz}

Contributors YS drafted the commentary and $\mathrm{MO}$ and YS revised and approved the final version to be published.

Funding YS is recipient of grants from the Spanish Ministry of Science and Innovation (MICINN) (PID2020119536RB-100 and PDC2021-121659-100), Valencian Agency of Innovation (INNVA1/2021/32) and the European Commission H2020 Programme through the EarlyCause Project (grant agreement: 848158). MO is a beneficiary of a European Marie Skłodowska-Curie Action of H2020 (grant agreement: 890545).

Competing interests YS is inventor of several patents on the application of intestinal bacteria to combat obesity (PCT/ES2013/070309; PCT/ ES2019/070821; PCT/ES2019/070882).

Patient and public involvement Patients and/or the public were not involved in the design, or conduct, or reporting, or dissemination plans of this research.

\section{Patient consent for publication Not required.}

Ethics approval This study does not involve human participants.

Provenance and peer review Commissioned; internally peer reviewed.

\section{(2) OPEN ACCESS}

Open access This is an open access article distributed in accordance with the Creative Commons Attribution Non Commercial (CC BY-NC 4.0) license, which permits others to distribute, remix, adapt, build upon this work non-commercially, and license their derivative works on different terms, provided the original work is properly cited, appropriate credit is given, any changes made indicated, and the use is non-commercial. See: http:// creativecommons.org/licenses/by-nc/4.0/.

(c) Author(s) (or their employer(s)) 2022. Re-use permitted under CC BY-NC. No commercial re-use. See rights and permissions. Published by BMJ.
Check for updates

To cite Sanz Y, Olivares M. Gut Epub ahead of print: [please include Day Month Year]. doi:10.1136/ gutjnl-2021-326781

Received 23 January 2022

Accepted 24 January 2022

\section{SLinked}

- http://dx.doi.org/10.1136/gutjnl-2021-325753

Gut 2022;0:1-2.

doi:10.1136/gutjpl-2021-326781

\section{ORCID iD}

Yolanda Sanz http://orcid.org/0000-0002-1615-1976

\section{REFERENCES}

1 Stefan N, Birkenfeld AL, Schulze MB. Global pandemics interconnected - obesity, impaired metabolic health and COVID-19. Nat Rev Endocrinol 2021;17:135-49.

2 Fan Y, Pedersen 0. Gut microbiota in human metabolic health and disease. Nat Rev Microbiol 2021;19:55-71.

3 Le Chatelier E, Nielsen T, Qin J, et al. Richness of human gut microbiome correlates with metabolic markers. Nature 2013;500:541-6.

4 Sze MA, Schloss PD. Looking for a signal in the noise: revisiting obesity and the microbiome. $\mathrm{mBio}$ 2016; 7:e01018-16.

5 Aron-Wisnewsky J, Prifti E, Belda E, et al. Major microbiota dysbiosis in severe obesity: fate after bariatric surgery. Gut 2019;68:70-82.

6 Rampelli S, Guenther K, Turroni S, et al. Pre-obese children's dysbiotic gut microbiome and unhealthy diets may predict the development of obesity. Commun Biol 2018;1:222.

7 Frost F, Kacprowski T, Rühlemann M, et al. Long-term instability of the intestinal microbiome is associated with metabolic liver disease, low microbiota diversity, diabetes mellitus and impaired exocrine pancreatic function. Gut 2021;70:522-30.

8 Berry SE, Valdes AM, Drew DA, et al. Human postprandial responses to food and potential for precision nutrition. Nat Med 2020;26:964-73.

9 Régnier M, Van Hul M, Knauf C, et al. Gut microbiome, endocrine control of gut barrier function and metabolic diseases. J Endocrinol 2021;248:R67-82.

10 Sanz Y, Romaní-Perez M, Benítez-Páez A, et al. Towards microbiome-informed dietary recommendations for promoting metabolic and mental health: opinion papers of the MyNewGut project. Clin Nutr 2018;37:2191-7.

11 Belda E, Voland L, Tremaroli V, et al. Impairment of gut microbial biotin metabolism and host biotin status in severe obesity: effect of biotin and prebiotic supplementation on improved metabolism. Gut 2022:9:325753

12 Voland L, Le Roy T, Debédat J, et al. Gut microbiota and vitamin status in persons with obesity: a key interplay. Obes Rev 2022;23:e13377.

13 Wu H, Tremaroli V, Schmidt C, et al. The gut microbiota in prediabetes and diabetes: a population-based crosssectional study. Cell Metab 2020;32:379-90.

14 Soto-Martin EC, Warnke I, Farquharson FM, et al. Vitamin biosynthesis by human gut butyrate-producing bacteria and Cross-Feeding in synthetic microbial communities. mBio 2020;11:e00886-20.

15 Peterson CT, Rodionov DA, Osterman AL, et al. B vitamins and their role in immune regulation and cancer. Nutrients 2020;12:3380. 